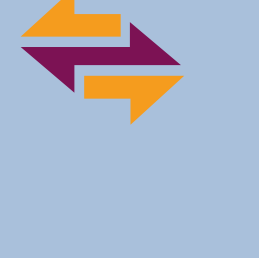

\section{Driving innovation with sports}

\author{
By Jan-Anders Mansson
}

\section{Introduction}

Sports have the ability to captivate people; they strive to display honest values, champion good sportsmanship, and, if done right, provide a safe, secure, and fair environment for those involved. Technological innovation is key in promoting healthy, fair, and safe sports. But, if left to its own devices, it can also lead to unequal access and increased risk for injury.

\section{The Olympic Agenda 2020 spirit}

In the world of sports, the Olympic Games are often regarded as the pinnacle. Governed by the International Olympic Committee (IOC), the Games provide a stage on which sports are tested and exhibited for the world to see. Therefore, the IOC has a responsibility to uphold a set of guidelines and values that its stakeholders should strive to meet.

The IOC's Agenda 2020 preaches a strong set of values. It includes 40 recommendations that are to serve as a strategic roadmap for the future of the Olympic Movement (see Figure 1). It ensures that the IOC is safeguarding the uniqueness of the Olympic Games and strengthening sports in society. Ideas for new products and projects often flow from a source of creativity, such as the Agenda 2020, which, in turn, can create new opportunities for a vast number of other fields, from mechanical engineering to architecture, and for society as a whole. In many different areas, new technologies, such as smart sensors, $5 \mathrm{G}$ compatible electronics, and lightweight materials, seep into this world, and their importance in sports and society alike cannot be understated.

Sports also have the unique ability to inspire healthy lifestyles in communities, countries, and even continents. They have helped mold societal structures by benefiting health organizations and inspiring grassroots movements. The ability of sports to influence and inspire society brings with it a responsibility to the sporting goods industry that should not be neglected.

New technology has to remain true to the values and rules of the sport, while also enhancing performance and entertainment. A sport should never be dictated by the technology that is used by the athletes; instead it should be proactive in deciding what technology is used and for what reason, with the goal of providing athletes with the tools necessary to perform at their best.

\section{Technology and sports}

Technology is used to improve sports performance, such as research and development for sport equipment and apparel. Often new materials and devices are released on the playing field well before they appear in other industries. Take, for example, carbon fiber composites that were initially developed for the aerospace industry, but found their way into bikes, tennis rackets, and skis long before they made a major impact on the aerospace industry. This turnover rate of the sportperformance market is surpassed only by consumer electronics. The implementation dynamics provides great opportunities for efficient technology exchange, with mutual benefits for the industrialization of new innovations.

Recent years have seen some major changes in digital technology and media, with a growth rate that can leave sports teams and organizations struggling to keep up (see Figure 2). Athletes use technology in training and competition, whether to increase performance or to

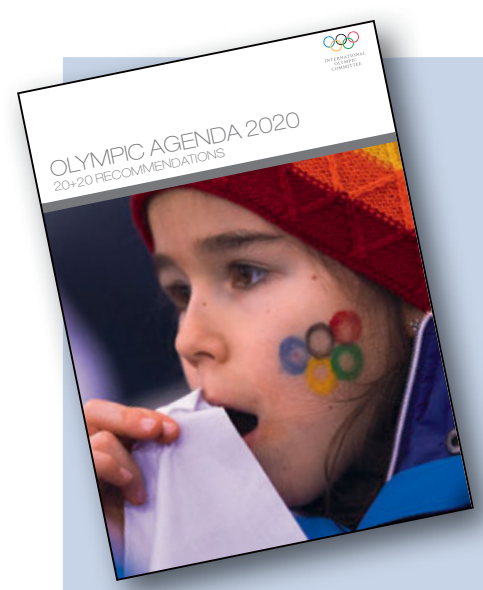

Figure 1. International Olympic Committee (IOC) Agenda 2020. Credit: IOC website.

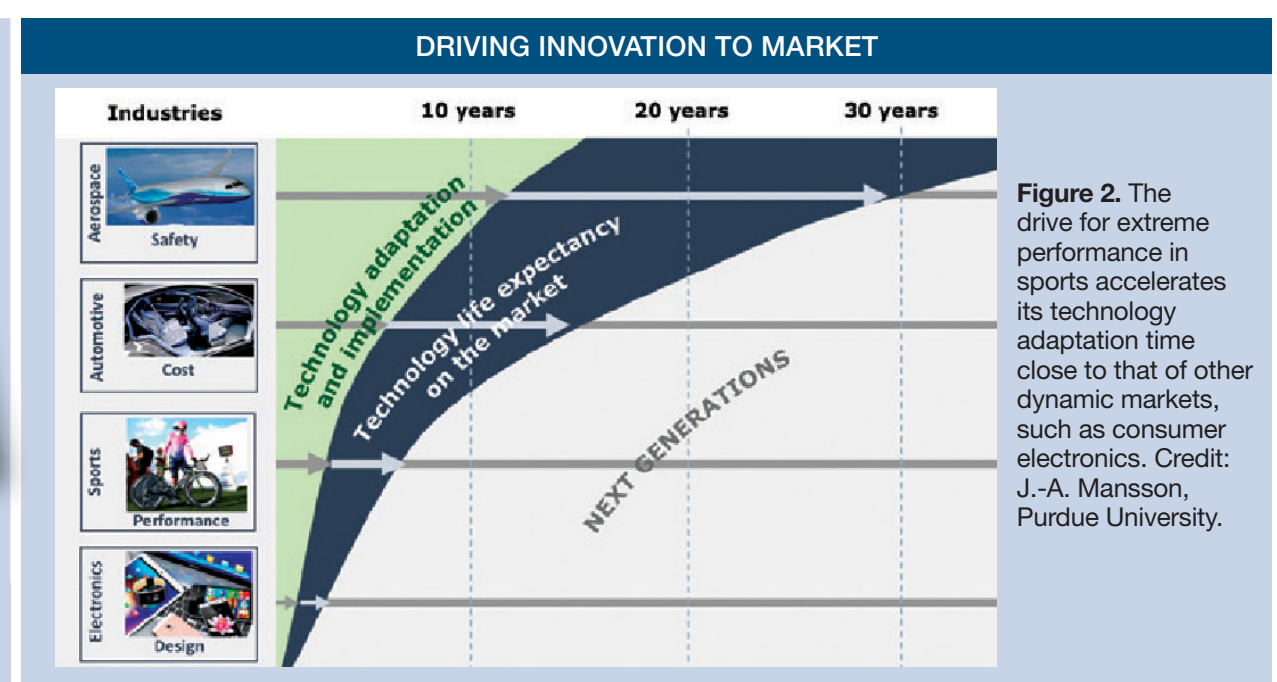


ensure their safety as external devices or integrated in their apparel. Wearable electronics such as heart rate monitors, smart GPS watches, and smart recovery sensors have been growing in importance. New technologies are also found in high-resolution cameras to show super slow-motion replays; drones carry cameras that offer distinct viewing angles; and digitally assembled footage enhances the viewing experience for spectators at home. Broadcast media and social media have revolutionized the way sports can be followed by the public. Information is moving faster, spreading wider, and making sports more intimate and personal.

\section{Innovation and safety}

The sports business is constantly drawn into a competition between athletes and spectators, competition and entertainment, safety and performance. The rise of alternative sports has pushed this balance closer to the edge. The use of new technologies, while they can benefit both the performance and safety of the athletes, can also become a danger if not managed correctly. In some cases, they require higher skill levels from the athletes which in turn increases the risk for injury. For example, carbon fiber bobsleds have led to high speeds on courses often not

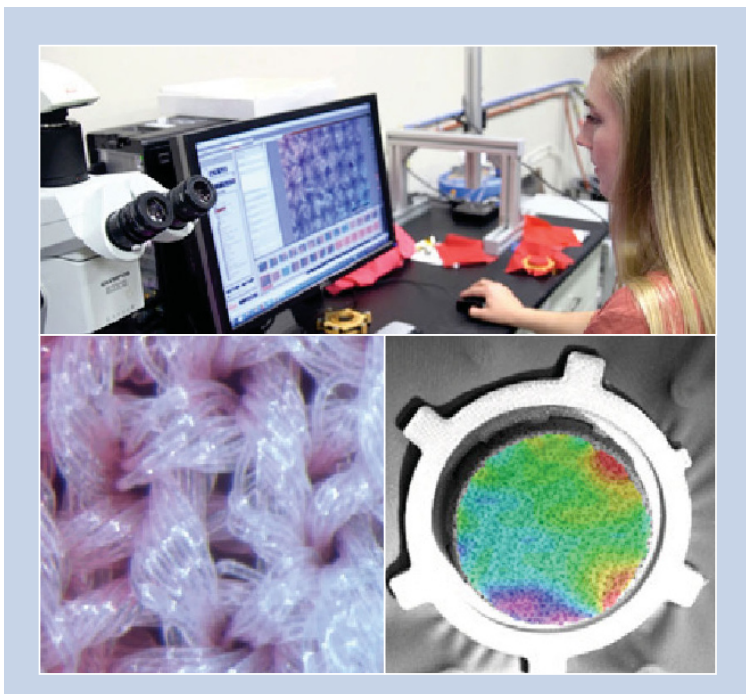

Figure 4. Swimsuit construction and materials are approved annually for competition based on permeability, surface coatings, dimensions, and construction to ensure a level playing field. Credit: J.-A. Mansson, Purdue University.
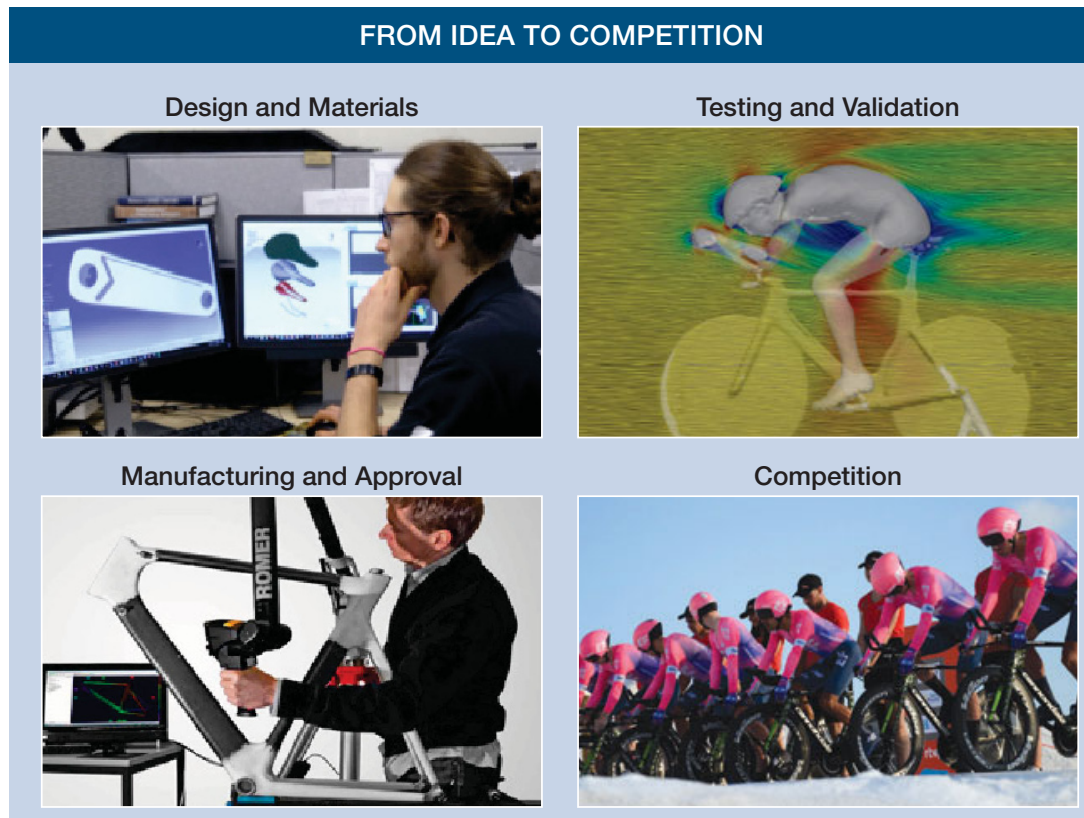

Figure 3. Today's competition bikes are examples of extremely fine-tuned composite technologies designed in harmony with the riders for performance and safety. Credits: (Top, left to right) Purdue University, Phil White; (Bottom, left to right) Hexagon AB, EF Pro Cycling.

suited for these higher speeds. A similar trend occurred in cycling, where the rise of stiffer and harder-to-handle bikes has coincided with higher speeds and greater injury risks (see Figure 3).

Has security and safety taken a back seat to the search for new technologies? Serious accidents or forms of cheating can negatively affect the reputation of sports. The responsibility for a clean stage comes down to all of us: athletes, international federations, event organizers, sponsors, managers, and parents. We need to ensure that the next generation can appreciate the positive values that sports can bring while remaining safe.

\section{Managing sports innovation and excitement}

A dimension that is often forgotten is what happens behind the stage - those managing the sports and trying to fulfill certain standards and implementing rules that allow the sport to be safe, clean, and fair.

Side effects and dangers that stem from rapid evolution should not be neglected. Proactive and responsible management is needed to safeguard a prosperous evolution. The constant appearance of novel technologies in competitive sports puts the managing federations and organization in a difficult spot. They are required to think about both positive and negative effects that new technologies might have on the sport, but they also have to be able to anticipate and proactively take measures to ensure the safety of the athletes and the accessibility of the sport to the masses. This was an issue the world of swimming faced with the introduction of the polyurethane swimsuit (see Figure 4) before the 2008 Olympic Games. During the following year's World Championships in Rome, more than 40 world records fell as a result of these suits, and drastic actions had to be taken by the governing body, FINA, to restore balance in the sport. Avoiding issues like this one takes careful planning by governing bodies, but they also have to be careful to not be seen as hindrances to technological progress. 
Managing sports is a challenging balance of ensuring that it remains exciting for a public with extremely high demands, while maintaining a certain level of tradition; allowing the use of modern and high-tech equipment without losing the respect for a level playing field during competition (cost, accessibility, availability); and driving performance without compromising safety and security.

\section{The Ray Ewry Sports \\ Engineering Center}

The Ray Ewry Sports Engineering Center at Purdue University works to address all of these topics, who they impact, and how they might be tackled. The Center remains at the forefront of discussions surrounding sports technology by taking various issues that arise around sports technology, and boiling them down into their essential generic engineering and scientific principles. The Center also serves as a resource for various stakeholders in sports when decisions need to be addressed surrounding the entrance of new technologies.

The Center announced a joint effort between the College of Engineering and Purdue Intercollegiate Athletics, which will reflect Ewry's passion for both sports and engineering and will advance research and learning benefits to athletes and students. The Center benefits Purdue student athletes and other high-level athletes in regional, national, and international competitions. Under an agreement with the IOC, the Center will provide a periodic newsletter reflecting on the "Latest and Brightest in Sports Technology."

The Center provides focus and energy to the groundbreaking and innovative research across Purdue that can impact athletic performance, safety, and the optimization of data science and sports engineering.

\section{Smart performance and fan experience}

Sensor-based technology is a rapidly expanding opportunity to make athletes more competitive while also enhancing the fan experience. The development of new sensors, along with the rise of $5 \mathrm{G}$ is going to lead to a revolution in the way information is used in sports. More sensor locations and lower latency will lead to increased opportunities for broadcasting
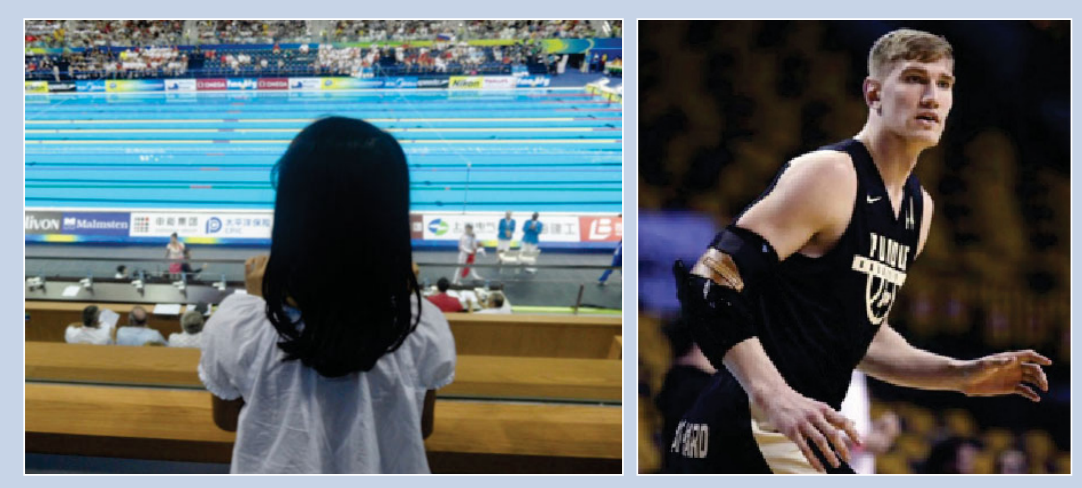

Figure 5. (Left) To ensure the healthy evolution of a sport, it is crucial to capture the mind of the next generation in the fan experience. Credit: J.-A. Mansson, Purdue University. (Right) Competition at the highest level requires the latest technology in preventative and rehabilitation devices. Credit: Purdue University Athletics.

and fan experiences while enhancing athletic performance. The Center is developing novel sensor-integrated versions of sports equipment to help athletes and coaches improve sports performance, training, and competition. Smart Stadium technology also offers extensive opportunities for enhancing fan engagement. The team is developing low-risk, high-return methods for optimizing Purdue athletes' performance, increasing fan interaction, and enhancing overall viewer excitement.

\section{Injury reduction and advanced rehabilitation}

Purdue is engineering solutions to decrease the likelihood of injury through the use of advanced protective equipment such as smart helmet technology and the design of safer playing surfaces. The Center's commitment to injury reduction and advanced rehabilitation serves as a valuable recruitment tool as Purdue continues to draw competitive student athletes. The Center is helping players who are undergoing rehabilitation to return to the field as safely and successfully as possible with noninvasive methods for quantifying the strength of healing tendons, ligaments, and bones, as well as designing new types of protective equipment and braces (see Figure 5).

\section{Sports integrity, fairness, and social integration}

Sports are far more than just physical activity. They build self-esteem, stimulate mental and emotional growth, relieve stress, and enhance a person's sense of accomplishment and social well-being. Significant new research addresses issues in the merging interfaces of sports integrity, fairness, and social integration. Innovative digital-based sport execution systems, enabled by new sensor and Internet of Things (IoT) technologies, in combination with miniaturization of devices, provide huge opportunities for next-generation sports management systems when seeking enhanced integrity and fairness. Monitoring and managing a level playing field will rely heavily on the development of smart and safe new technologies in the future.

The Ray Ewry Sports Engineering Center at Purdue University

Team

Jan-Anders E. Mansson, Director Distinguished Professor of Materials and Chemical Engineering

\section{Eric Nauman}

Professor of Mechanical Engineering

Nathan W. Hartman

Dauch Family Professor of Advanced

Manufacturing

Founding Members

Mung Chiang

John A. Edwardson Dean of the College of Engineering

Mike Bobinski

Vice President, Director of Intercollegiate Athletics 